IEEE Transactions on Image Processing

vol. 38, pp. 2127-2136, September 2009

\title{
Personal Authentication using Hand Vein Triangulation and Knuckle Shape
}

Ajay Kumar, K. Venkata Prathyusha

This paper presents a new approach to authenticate individuals using triangulation of hand vein images and simultaneous extraction of knuckle shape information. The proposed method is fully automated and employs palm dorsal hand vein images acquired from the low-cost, near infrared, contactless imaging. The knuckle tips are used as key points for the image normalization and extraction of region of interest. The matching scores are generated in two parallel stages; (i), hierarchical matching score from the four topologies of triangulation in the binarized vein structures and (ii) from the geometrical features consisting of knuckle point perimeter distances in the acquired images. The weighted score level combination from these two matching scores are used to authenticate the individuals. The achieved experimental results from the proposed system using contactless palm dorsal-hand vein images are promising (equal error rate of $1.14 \%$ ) and suggest more user friendly alternative for user identification.

\section{Introduction}

The rapid growth in the use of e-commerce applications requires reliable user identification for effective and secure access control. The face identification beyond visible spectrum is increasing receiving attention and has highest user acceptance [23], [7]. Hand vein identification has emerged as a promising component of biometrics study. The subcutaneous vascular pattern/network appearing on the back of hand, referred to as the hand vein in this paper, is extremely difficult to forge and therefore offers promising biometric which also ensures liveness. The figure 1 illustrates the generic vascular map found on the dorsum of the hand. There are mainly two types of hand veins found on the dorsum of the hand, namely cephalic and basilic. The basilic veins are the group of veins 
attached with surface of hand and marked as ' 2 ' in figure 1 . It generally consists of upper limb of the back of hand. Cephalic veins are the group of veins attached with the elbow of the hand and marked as ' 1 ' in figure 1 . The vascular system extends or shrinks with the respective changes in the body. The shape of hand veins changes with the change in the length of body from childhood. In general, no major growth takes place in the adult life and hence vein patterns are quite stable in the age group of 20-50. After that the vascular system begins to shrink with the decline in the strength of bones and muscles. These changes in vascular system make the vein pattern loose and change its size as compared to earlier. As the vascular system is a large and essential system of the body, it is largely affected due to any change in body; either by nature or by disease. The diabetes, hypertension, atherosclerosis, metabolic disorders [25] and tumors [26] are some diseases which affect the vascular systems and made it thick or thin. There have been several other efforts to investigate the utility of hand vein patterns for effective user authentication. In the following section, we present a brief review on the related prior work which is followed by the salient features of the proposed approach investigated in this paper.

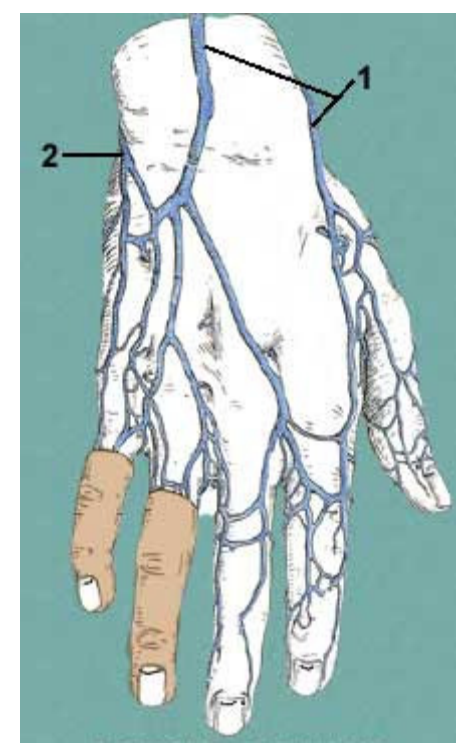

Figure 1: Example of the generic vascular map on the palm dorsal surface (courtesy of [24]). 


\subsection{Prior Work}

The stability and uniqueness of hand vein patterns have attracted the attention of researchers for its usage in the personal identification. The thermal imaging of palm dorsal surfaces typically captures the thermal pattern generated from the flow of (hot) blood in cephalic and basilic veins. Lin and Fan [1] have investigated the personal verification from such palm dorsal images acquired from the thermal infrared (IR) camera operating in $(3.4-5) \mu \mathrm{m}$ range. The approach detailed in [1] is fully automated and uses the combination of multiresolution representations from the post processed thermal vein patterns. Wang and Leedham [2] present yet another approach for personal authentication using hand vein images acquired from the thermal imaging. Authors [2], [22] have employed Hausdorff distance to generate matching scores between the extracted line patterns and illustrated promising results.

The subcutaneous blood vessels absorb less radiation, in near IR (780-1100 nm) illumination, than its surrounding blood and therefore generates high contrast in the acquired images. Such near IR imaging of hand vein patterns have also been investigated in the literature for personal identification. Cross and Smith [3] have detailed the usage of near IR imaging for the extraction of hand vein patterns. Authors have demonstrated the two-fold matching of medial axis representation, following the vein skeleton extraction, for the authentication of 20 users. Im et al. [4]-[5] have presented the implementation for hand vein extractor, using FPGA in [4] and DSP processor in [5], but with little details on the matching strategy or on the size/nature of database employed for the performance evaluation. Tanaka and Kubo [8] also developed hand vein acquisition device using near IR imaging and employed FFT based phase correlation scheme for user verification. 
Recently some commercial products, e.g. [36], [37], that authenticate individuals from hand veins images are also available. Personal authentication using conjunctival vasculature pattern has been reported in [38].

It is widely known that the thermal (far IR) imaging cameras are highly sensitive to ambient conditions and very expensive. Therefore the researchers [4]-[5], [8], [10] have focused on the solutions using near IR imaging. The imaging setup in prior work [3]-[4], [8], [13]-[14] employed hand docking frame device (to restrict the translational and rotational changes) which are often inconvenient and not user friendly. Furthermore, with the notable exception of [1], [3], there is no systematic (completely automated) proposal for hand vein based user authentication.

\subsection{Proposed System}

In this paper, we develop a new hand vein authentication approach utilizing the structural similarity of hand vein triangulation and knuckle shape features. The block diagram of the proposed approach is shown in figure 2. The approach has been adapted to utilize palm dorsal images acquired from the, low-cost, contactless, near IR imaging. The main contributions from this paper [33] can be summarized as follows:

(i) In this paper, we investigate the extraction and matching of hand vein structure using the key point triangulation. It many be noted that the low quality (visibility and clarity) of vein images does not guarantee same number of key points (from the same user) and therefore a weighted combination of four different types of triangulation is developed. Further details of this strategy can be seen section VII.

(ii) The proposed method also investigates the utility of knuckle shape features since these features can be simultaneously extracted from the acquired images. The matching 


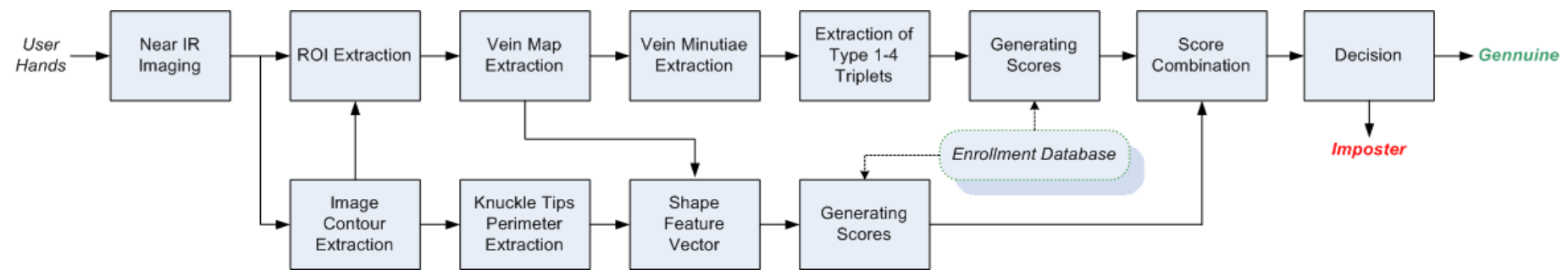

Figure 2: Block diagram of the personal authentication using near IR hand vein images.

scores computed from these knuckle shape features are employed to further improve the performance for the hand vein authentication.

(iii) The usefulness of the proposed method is investigated from the experimental results on the real hand vein images acquired using contactless near infrared imaging. The contactless imaging is expected to yield higher image variations. Therefore the steps of image normalization and feature extraction have been adapted to reduce the variations in the interclass matching scores.

The image contours extracted from the acquired images are used for the image normalization and segmentation of region of interest (ROI) which is detailed in section II-IV. The automated extraction of hand vein map from ROI images is described in section $\mathrm{V}$. The extraction and triangulation of local key points from the hand vain map is detailed in section VI and section VII respectively. The hierarchical matching scheme for the triplets is introduced in section VIII. The experiments and results from this work are presented in section IX which is followed by the discussion in section $\mathrm{X}$ and the main conclusions from this paper are summarized section XI.

\section{Image Acquisition}

The acquisition of hand vein images using near IR imaging has been studied in [3], [11], [15]. In this work, a low-cost near IR camera, traditionally used for surveillance, was employed for the contactless image acquisition. The near IR illumination (LED's) is 
evenly and circularly located around the camera and peaks at $850 \mathrm{~nm}$ wavelength ${ }^{*}$. The employed near IR camera [12] is fixed at the base and shown in figure 3(a). The expected distance between the user hand and the camera was experimentally fixed to $21 \mathrm{~cm}$ for the best case acquisition. The volunteers were requested to present their folded right hand (palm dorsal surface) near the imaging window such that the knuckle tip from the middle finger remains at the top. The acquisition of a typical contactless hand vein image is shown in figure 3(b). Some of the image samples acquired from our imaging setup are reproduced in figure 4.
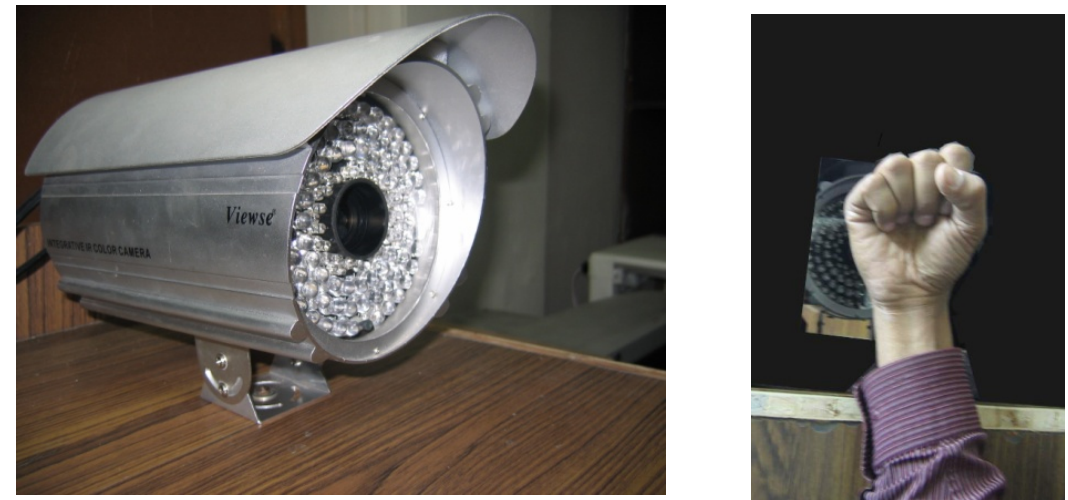

Figure 3: The image acquisition setup; (a) employed near IR camera, (b) contactless image acquisition.
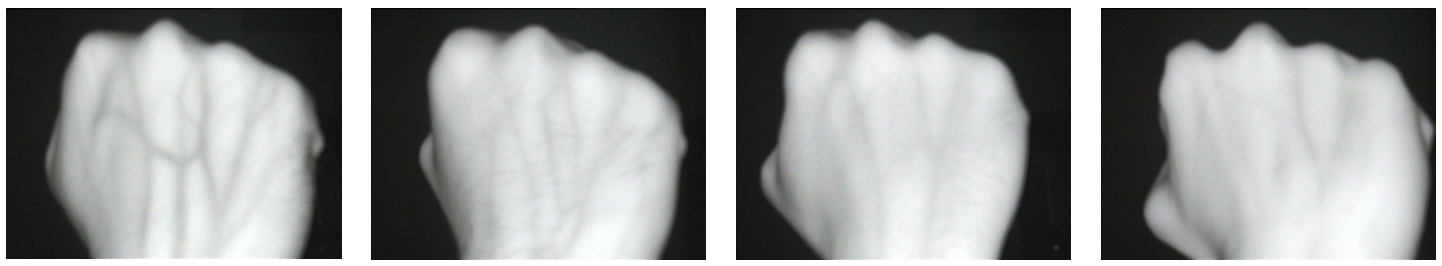

Figure 4: Samples of the acquired hand vein images.

The incident near-infrared illumination travelling in the palm dorsal skin is absorbed by the haemoglobin in the blood and branches of artery and vein. In addition to the different absorption coefficients, the scattering coefficients of blood and bio-tissue for near infrared illumination are significantly different [34]. The higher scattering coefficient of

\footnotetext{
* This wavelength lies in the medical spectral window $(700-900 \mathrm{~nm})$ in which the illumination penetrates deeper into the tissues [17].
} 


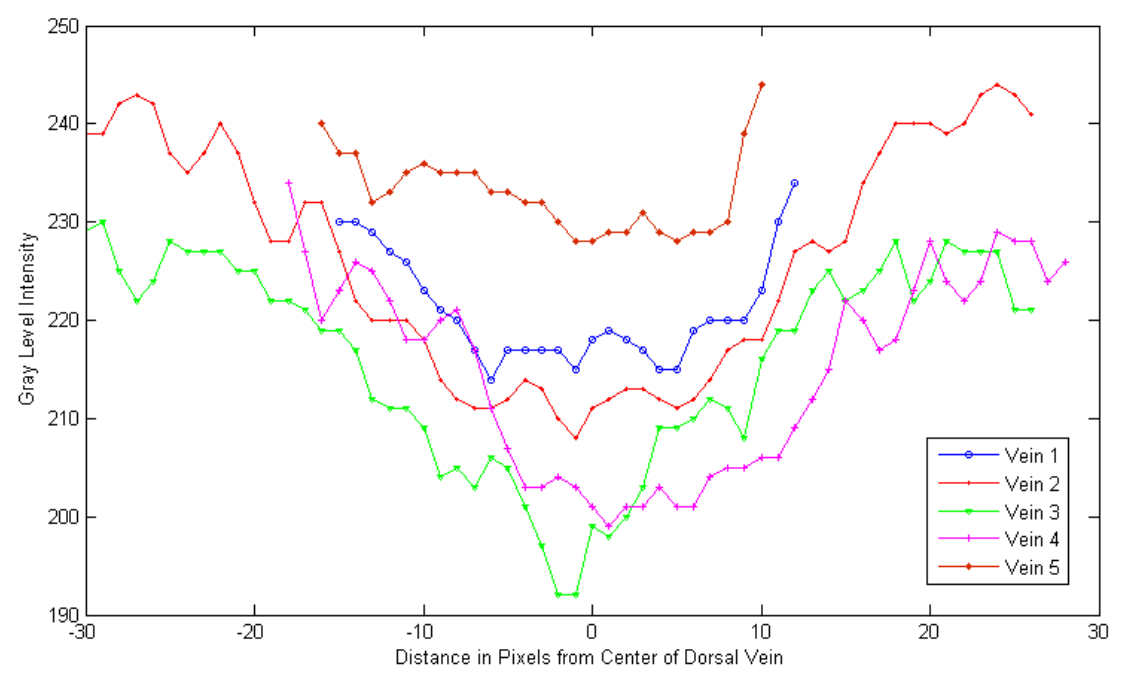

Figure 5: The gray-level profiles of the cross section of blood vessels in figure 4 (first image).

the blood ensures that more incident infrared illumination changes its path in blood than in the surrounding tissue. It is scattering rather than absorption that dominates and results in the darker appearance of dorsal vein patterns. The figure 5 shows representative samples of gray level vein profiles along the direction perpendicular to their length. It can be observed from this figure that these vein profiles are quite noisy and do not have any ideal step edge to distinguish from the background. Although these intensity patterns varies from vein to vein, their average profile can be approximated as Gaussian. The widths of these vein profiles are found in the range of 30-50 pixels. The edge point of vein patterns can be detected by locating zero crossings of second derivative gray level profiles. However, the derivative filters are very sensitive to the accompanying noise (figure 5). Therefore it is judicious to employ some pre-processing, such as averaging, to reduce the effect of noise. This two step process (Laplacian of Gaussian) was found to be quite effective for the localization of venous structure in the acquired hand vein images.

\section{Extraction of Knuckle Tips}

The acquired images are firstly binarized using Otsu's thresholding and its contour is obtained as shown in figure 6 (a). One of the key tasks in image normalization is to 
obtain reliable control points from the image that can be used for alignment and extraction of region of interest (ROI). In our approach, we selected knuckle tips as the control points. The knuckle tips can be easily extracted by locating the peaks in the distance from of every contour point from the middle base point $C_{b}$, similar to the method used for locating finger tips in the hand geometry identification [19]-[20]. Another simpler method is to scan the contour image from left to right and from top to bottom. The point where the first transition occurs from white to black pixel is the first knuckle tip $K_{\mathrm{m}}$. Similar scanning of the two portions of the image, i.e. left side and right side of $K_{\mathrm{m}}$, can locate index finger tip $\left(K_{\mathrm{i}}\right)$ and ring finger tip $\left(K_{\mathrm{r}}\right)$. However, as can be observed from figure 4, 6 (or other images in this paper) the little finger knuckle tip (say $K_{1}$ ) is usually not visible and difficult to locate. In our implementation the point $K_{1}$ is approximated contour point at the same row and column distance, as between $K_{\mathrm{m}}$ and $K_{\mathrm{r}}$, from knuckle tip $K_{\mathrm{r}}$ but from the aligned image contour.

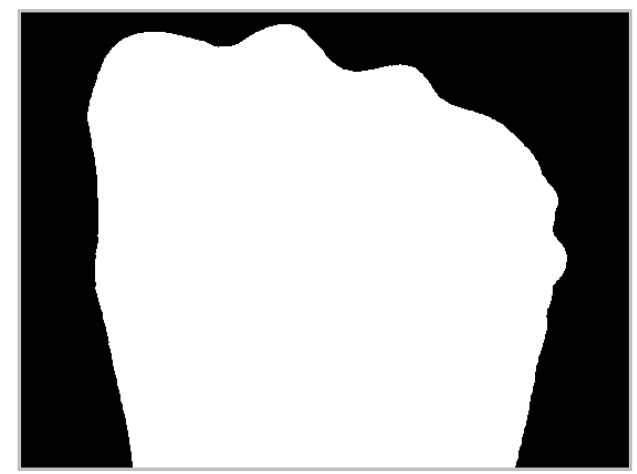

(a)

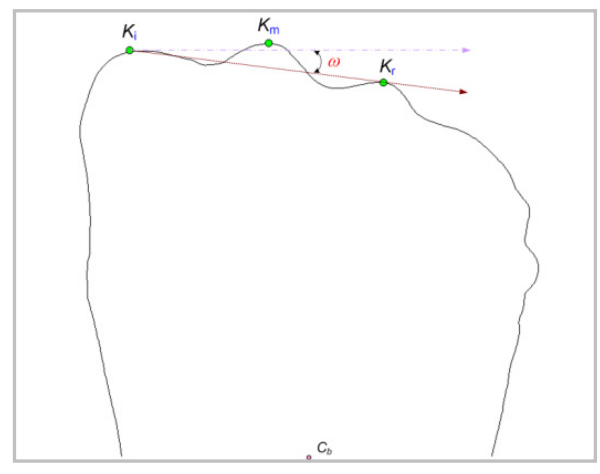

(b)

Figure 6: (a) The acquired image after thresholding and (b) corresponding contour image with extracted key points for the normalization.

\section{Extraction of Region of Interest}

Once the key control points $\left(K_{\mathrm{i}}, K_{\mathrm{m}}, K_{\mathrm{r}}\right)$ are located, the image orientation is firstly ascertained to extract reliable region of interest. The angle $\omega$, as shown in figure 6 (b), is 
firstly computed. The rotation matrix $\Omega$ is used to achieve the vertical alignment of the region of interest, from each of the acquired image, as follows:

$$
\Omega=\left[\begin{array}{rr}
\cos (\omega) & -\sin (\omega) \\
\sin (\omega) & \cos (\omega)
\end{array}\right], \quad \omega=\tan ^{-1}\left\{\frac{K_{r}(y)-K_{i}(y)}{K_{r}(x)-K_{i}(x)}\right\}
$$

where $(x, y)$ represents the corresponding coordinates of knuckle tips. Once the alignment angle is estimated, a fixed region of interest (ROI) of size $400 \times 300$ pixels is extracted. The size and position of this region is empirically selected and fixed at 150 pixels below $K_{\mathrm{m}}$ and along the line joining $K_{\mathrm{i}}$ and $K_{\mathrm{r}}$.

\section{Extraction of Vein Structure}

The topological structure of vein patterns is extracted from the segmented region of interest. The images acquired from the low-cost near IR camera are of poor quality and contains obscure parts of veins, mainly due to noise and uneven illumination profile of near IR illuminators fixed at distance. Therefore the method of vein pattern extraction is empirically tailored to the nature of acquired images and is summarized in figure 7 . Firstly the images are subjected to adaptive histogram equalization for image enhancement using local gray level information. The enhanced images are further processed using Laplacian of Gaussian (LOG) or Mexican hat operator.

$$
\nabla^{2} G_{\sigma}(x, y)=-\frac{1}{\pi \sigma^{4}}\left\{1-\frac{x^{2}+y^{2}}{2 \sigma^{2}}\right\} e^{-\frac{\left(x^{2}+y^{2}\right)}{2 \sigma^{2}}}
$$

The LOG operator $\nabla^{2} G_{\sigma}(x, y)$ is employed to identify the image regions with rapid intensity changes while suppressing the noise. The resulting image is subjected to thinning which generates the vein pattern structure. However, the resulting binary pattern 
also contains noise, i.e. isolated regions with small connectivity. Therefore the isolated regions with small (empirically determined threshold) amount of connectivity are eliminated from the thinned hand vein pattern images.

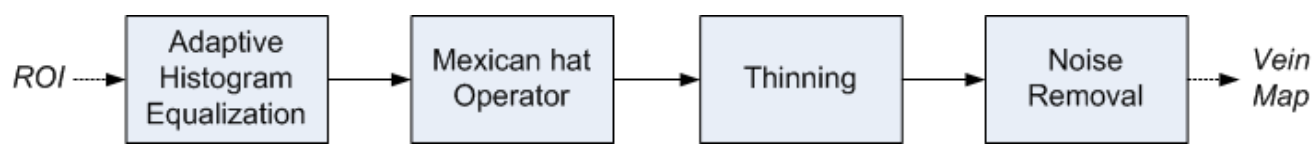

Figure 7: Extraction of hand vein map from the region of interest images.

\section{Extraction of Vein Bifurcatioin and Vein Endings}

The individuality of vein structure is exclusively determined from the relationship among the local vein characteristics. Therefore the extracted vein pattern is firstly used to locate the key points that are relatively stable, unique and repeatable. The vein bifurcation and endings points are selected as key points to extract local vein properties. A vein bifurcation is defined as vein point where vein forks or diverges into branch veins, and the vein ending is the point at which vein ends or disappears abruptly. This disappearance could be due to the abrupt ending of blood vessels (as shown in figure 1) or their poor visibility from the imaging system. In order to extract the vein endings and bifurcation points we examining the connectivity of every pixel and determine the crossing number $R(\mathbf{m})$ [16] for every pixel $\mathbf{m}$. The crossing number $R(\mathbf{m})$ is the sum of differences between pairs of adjacent pixels in $3 \times 3$ window centered at $\mathbf{m}$.

$$
R(\mathbf{m})=\sum_{z=1}^{8}\left|\operatorname{val}\left(\mathbf{m}_{z \bmod 8}\right)-\operatorname{val}\left(\mathbf{m}_{z-1}\right)\right|
$$

The pixel $\mathbf{m}$ with $\operatorname{val}(\mathbf{m})=1$ corresponds to vein ending point if $R(\mathbf{m})=2$, and corresponds to vein bifurcation point if $R(\mathbf{m}) \geq 6$.

\section{Feature Extraction Using Minutiae Triangulation}


The feature extraction approach is to use unique topological structure from the hand vein minutiae using Delaunay triangulation [21]. A minutiae $M_{\mathrm{i}}$ can be represented by its position and type, i.e. $M_{\mathrm{i}}=\left(p_{\mathrm{i}}, q_{\mathrm{i}}, m_{\mathrm{i}}\right)$ where $\left(p_{\mathrm{i}}, q_{\mathrm{i}}\right)$ denotes the position and $m_{\mathrm{i}}$ denotes the type of minutiae (vein bifurcation or endings). The idea is to extract meaningful

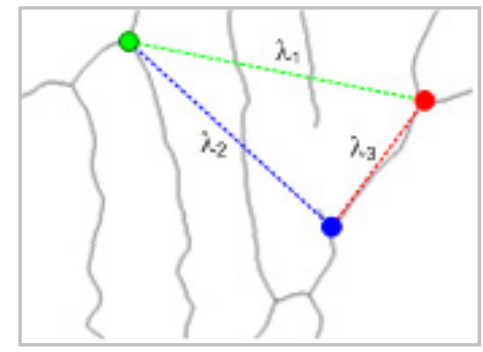

Figure 8: Selection of a triplet (type 1) from the extracted hand vein map.

minutiae groups, i.e., triplets or triangles, from the hand vein map to achieve rotation and translation invariant representation of the local information. Given a minutiae triangle (figure 8), we separately compute three lengths $\lambda_{1}, \lambda_{2}$, and $\lambda_{3}$. Then all the sides of triangle are sorted to avoid considering all possible orders of same lengths.

$$
\lambda_{1} \geq \lambda_{2} \geq \lambda_{3}
$$

A four dimensional index $\left(\varepsilon_{\mathrm{j}}, \lambda_{1}, \lambda_{2}, \lambda_{3}\right)$ with $\varepsilon_{\mathrm{j}}=1,2,3,4$, is formed by indexing the triplets in the vein map. As shown in table 1, we considered four types of triplets, i.e. $\varepsilon_{j}$, depending on the combinations of three types of minutiae. The number of minutiae points that can be extracted from the low quality ROI images is limited. In addition, the matching strategy should also accommodate missing and spurious minutiae points. We consider that two triplets $Y$ and $Y$ are matched only if they satisfy following set of conditions.

$$
\varepsilon_{j}=\varepsilon_{j},\left|\lambda_{1}-\lambda_{1}\right|<T_{\mathrm{m}},\left|\lambda_{2}-\lambda_{2}\right|<T_{\mathrm{m}},\left|\lambda_{3}-\lambda_{3}\right|<T_{\mathrm{m}}
$$


The threshold $T_{\mathrm{m}}$ can be empirically selected depending on the image size and quality.

Table 1: Hierarchal classification of triplet types

\begin{tabular}{|c|c|c|c|}
\hline Triplet Type & \multicolumn{3}{|c|}{ Minutiae Type $^{*}$} \\
\hline$\varepsilon_{j}$ & $m_{1}$ & $m_{2}$ & $m_{3}$ \\
\hline 1 & $b$ & $b$ & $b$ \\
\hline 2 & $b$ & $b$ & $e$ \\
\hline 3 & $b$ & $e$ & $e$ \\
\hline 4 & $e$ & $e$ & $e$ \\
\hline
\end{tabular}

$* b$ - bifurcation, $e$ - ending

\section{Score Assignment}

Once the extracted triplets are matched, using the criteria in equation (5), the matching scores are assigned. The score assignment scheme is hierarchical and assigns higher scores to more likely true matches. If two triplets having three bifurcation points, i.e. type 1 , are matched then there is higher probability that they corresponds to the same user vein map. Therefore such matches are assigned higher scores. However, those matching triplets formed due to three vein endings, i.e. type 4, have small probability/reliability that they have originated from the vein map of the same user. Our observations have

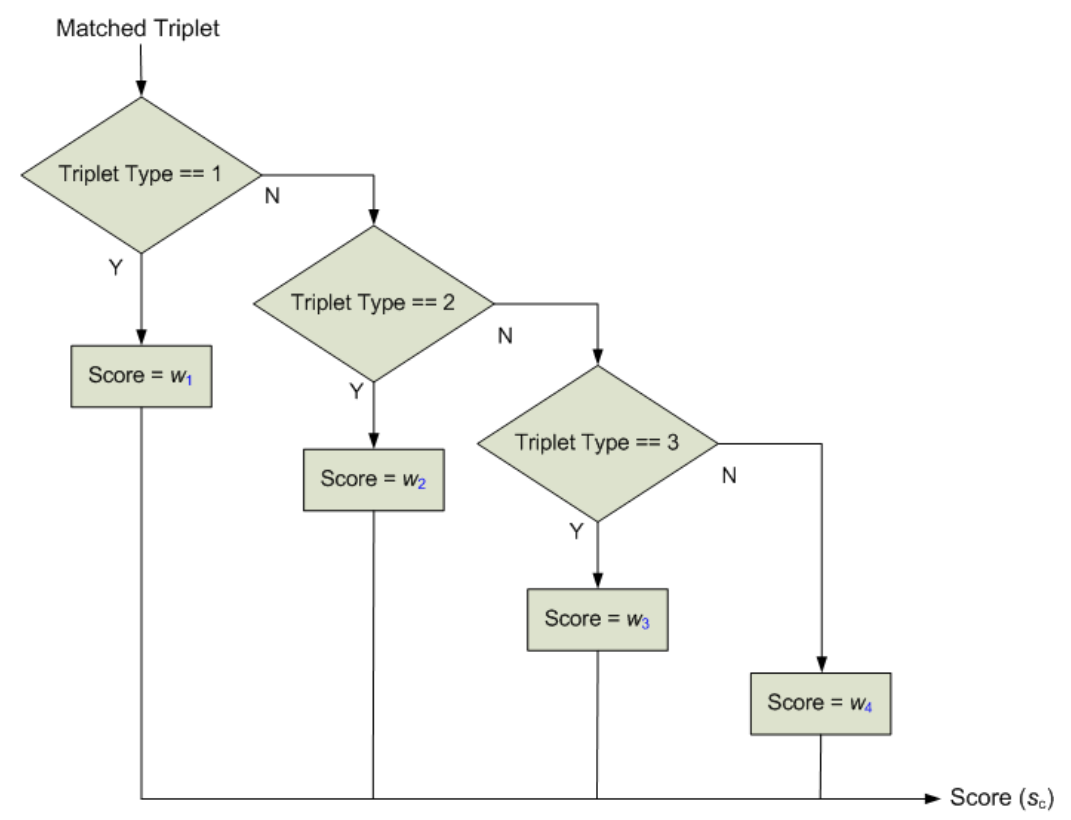

Figure 9: Score generation from a matched triplet. 
suggested that the chances of spurious vein ending formation (due to image quality or vein extraction process) in vein map are higher. Therefore the score assignment for such matched triplets is the lowest (figure 9). The weights (or scores) are experimentally determined and such that $w_{1}>w_{2}>w_{3}>w_{4}$. Thus each of the matched triplet type is assigned a score and the cumulative matching score $\left(s_{\mathrm{c}}\right)$ from all the matched triplet types is computed for every pair of hand vein matching. In addition to the usage of matching scores from the vein map (as discussed above), we also investigate the usefulness of additional features that are simultaneously extracted from the acquired images. The perimeter of pixels between four knuckle tips $\left(K_{\mathrm{i}}, K_{\mathrm{m}}, K_{\mathrm{r}}\right.$, and $\left.K_{\mathrm{l}}\right)$ from the normalized contour image is computed. Thus three contour distances, say $p_{1}, p_{2}, p_{3}$ corresponding to distance between knuckle points $K_{\mathrm{i}}-K_{\mathrm{m}}, K_{\mathrm{m}}-K_{\mathrm{r}}, K_{\mathrm{r}}-K_{1}$ respectively are also used as features. In addition, the perimeter of vein map (extracted as detailed in section $\mathrm{V}$, figure 7), i.e. number of white or on pixels $\left(p_{4}\right)$ are also used for the characterization of vein shape. Thus four features $\left(p_{1}-p_{4}\right)$ are used to form separate shape feature vector. The Euclidean distance between two shape features, from the two hand vein images, is used as shape matching score $\left(s_{\mathrm{g}}\right)$. The weighted combination of two matching scores, $s_{\mathrm{c}}$ and $s_{\mathrm{g}}$, is used compute a single consolidated matching score [35]. These consolidated matching scores are used to assign class labels (genuine or imposter) using the decision threshold.

\section{Experiments and Results}

The performance of the proposed authentication scheme was evaluated on hand vein database acquired in the real environment. This database mainly consisted of the right hand vein images collected from the students and staff in our university. The database from the 100 users, with three images per user, was acquired during October 2006 to May 
2007. All the subjects in the database are in the age group 14-55 years comprising of 81 males and 19 females. The imaging setup has been detailed in section II and the size of each of the acquired images was $768 \times 576$ pixels. The peg-free imaging introduces scale changes as it is very unlikely that user presents his/her hand at the same distance during successive imaging. In addition, the acquired images also had rotational intra-class variations. Some volunteers were not very cooperative and none of them were paid for the imaging.

The ROI extraction steps detailed in section III and IV were employed to extract $400 \times 300$ pixels size ROI images. These images were used to extract vein map as detailed in section V. The standard deviation of the $60 \times 60$ Mexican hat operator was empirically fixed to 0.25 . The contrast limited adaptive histogram equalization was performed on $9 \times 9$ subregions and matched to Rayleigh distribution with the variance of 9. All the isolated regions with 8 connected pixels and less than 100 counts were removed from the thinned images (noise removal step in figure 7). Figure 10 and 11 shows samples

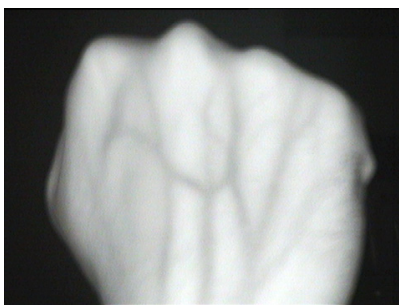

(a)

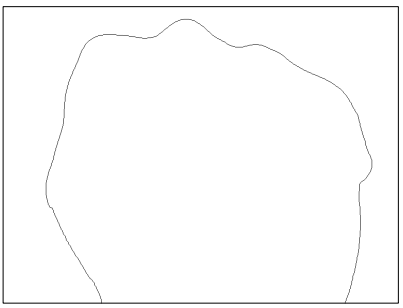

(c)

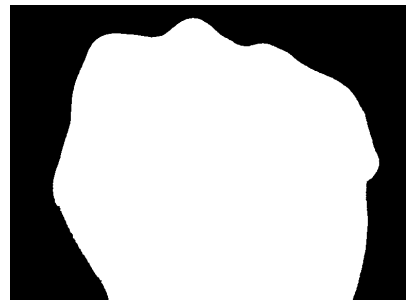

(b)

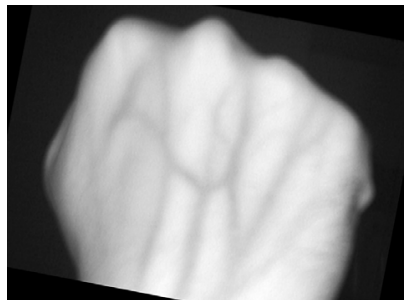

(d)

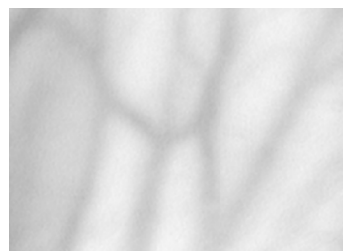

(e)

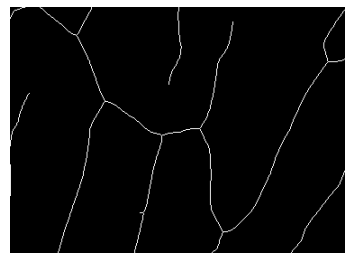

(f)

Figure 10: Extraction of vein topology, (a) Acquired hand images, (b) binarized image, (c) hand contour, (d) normalized image, (e) extracted region of interest, and (f) extracted hand-vein topology 


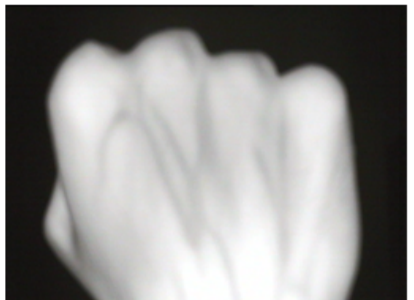

(a)

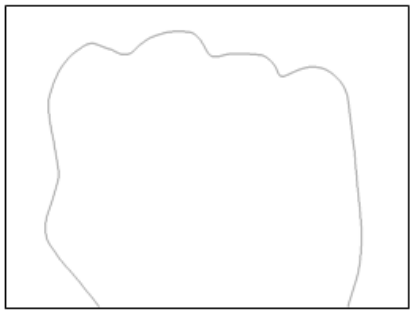

(c)

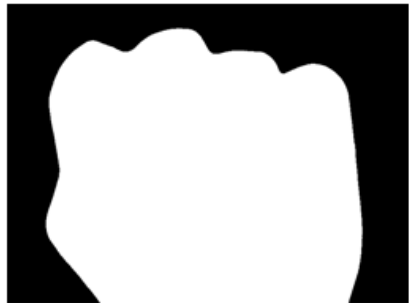

(b)

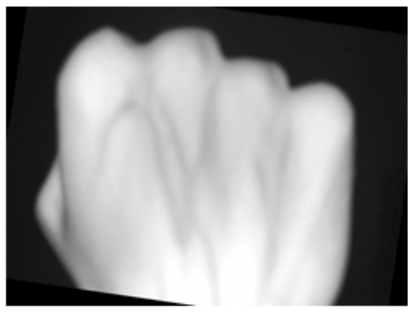

(d)

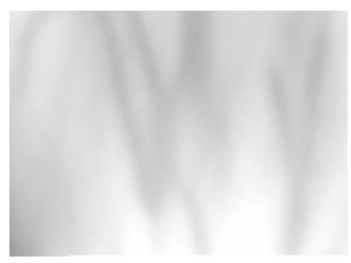

(e)

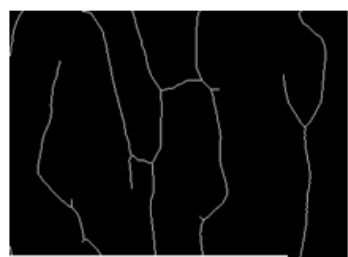

(f)

Figure 11: Extraction of vein topology, (a) Acquired hand images, (b) binarized image, (c) hand contour, (d) normalized image, (e) extracted region of interest, and (f) extracted hand-vein topology

from the acquired images, corresponding normalized images, extracted region of interest and their respective vein map. The number of vein bifurcation points that can be extracted from the acquired images (region of interest) never exceeded 10. However, the matching scheme detailed in section VII has been adapted to handle the spurious

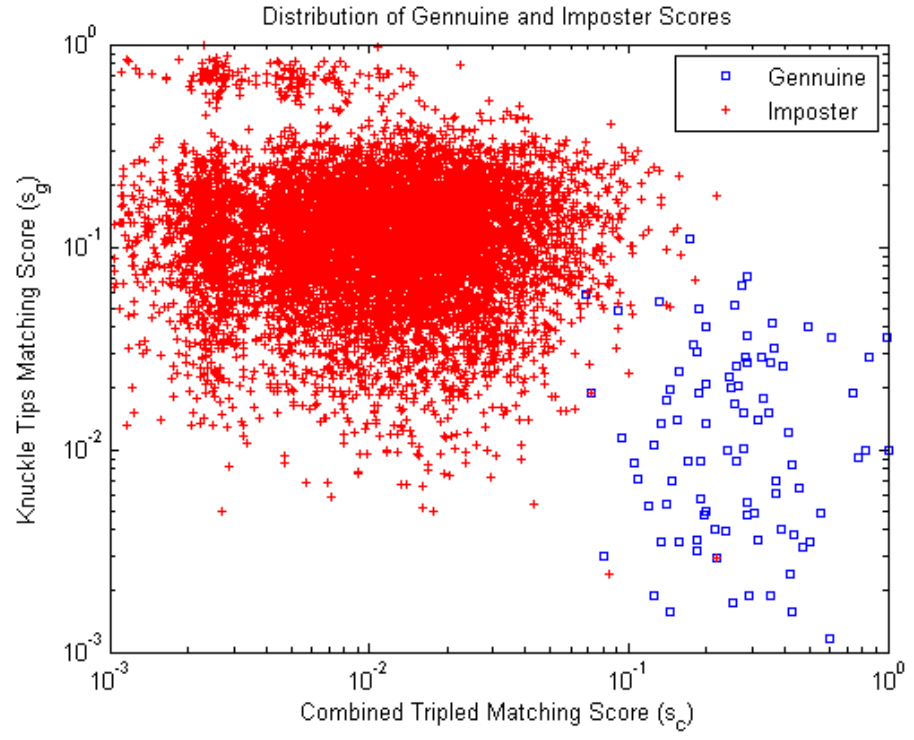

Figure 12: Distribution of genuine and imposter matching scores. 
vein endings. The weights from different triplet types were empirically selected as 40, 15, 8,3 corresponding to $w_{1}, w_{2}, w_{3}$ and $w_{4}$ respectively. The experimental results reported in this paper employed two images for the training and rest one to ascertain the test performance for the user authentication. However, this was repeated and the average performance from the three different test set is reported. We firstly evaluated the performance from matching scores using $\left(s_{\mathrm{c}}\right)$ which was generated from the triplet matches. Then the performance from the geometrical features obtained from the knuckle tip distances, i.e. matching scores $s_{\mathrm{g}}$, was integrated to ascertain the performance improvement. The weights for the combination of two matching scores, $s_{\mathrm{c}}$ and $s_{\mathrm{g}}$, were empirically selected as 0.8 and 0.2 respectively. The distribution of genuine and imposter scores obtained from the triplet matches and from the geometrical features is shown in figure 12. The receiver operating characteristics from the test data for the average performance is shown in figure 13. The performance from the usage of knuckle tip distances is very low. However, the performance improvement due to the integration of these features is significant and can be observed from the same figure. Figure 14 shows

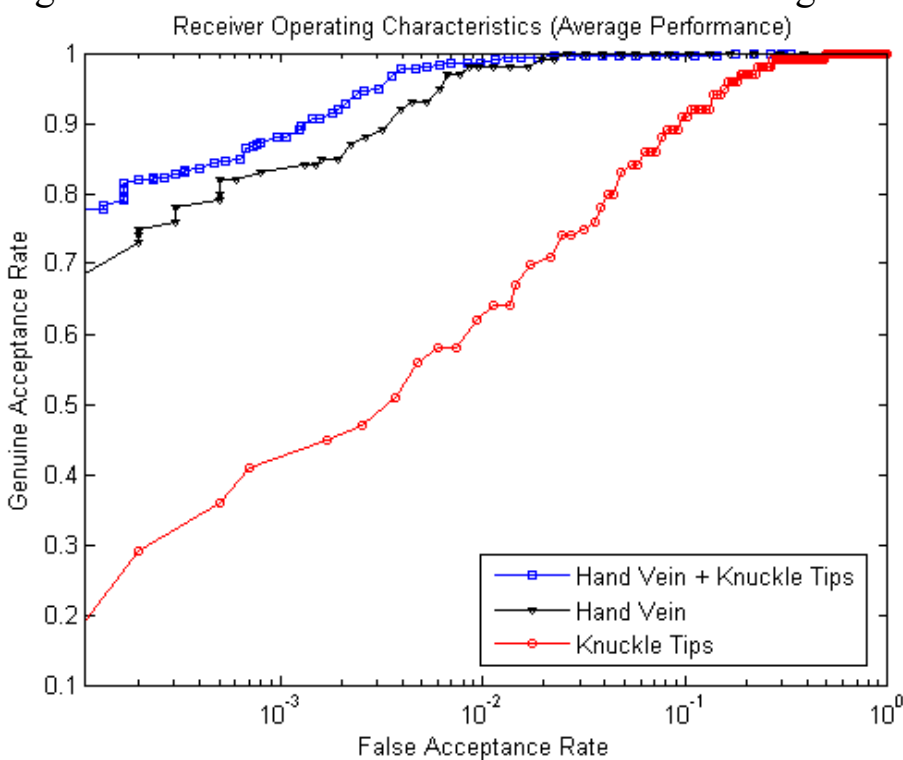

Figure 13: The ROC for the average performance from the test image samples. 


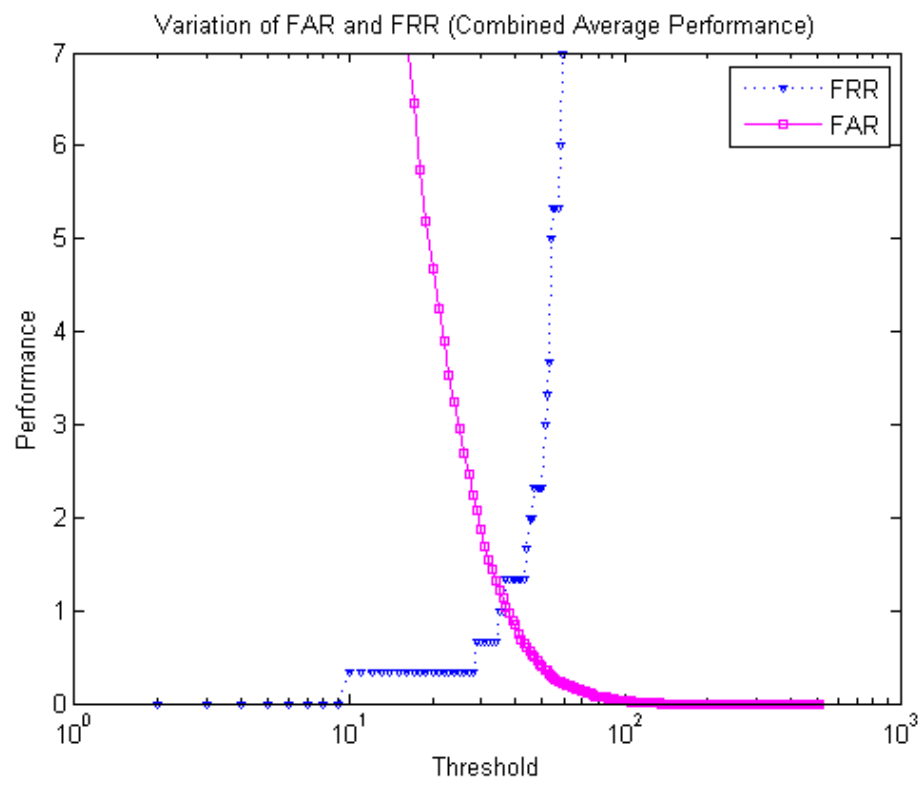

Figure 14: The variation of FAR and FRR with decision threshold (combined performance).

the variation of the False Acceptance Rate (FAR) and False Reject Rate (FRR) with the decision threshold, corresponding to the combined matching scores. The equal error rate from the matching scores due to triplets alone $\left(s_{\mathrm{c}}\right)$ is $1.77 \%$. The equal error rate of $1.14 \%$ was achieved from the combined matching scores which can also be seen from figure 14 .

Figure 15 (a) shows the distribution of combined triplet matching scores obtained from the palm dorsal veins for the genuine user comparisons. Similarly, the distribution

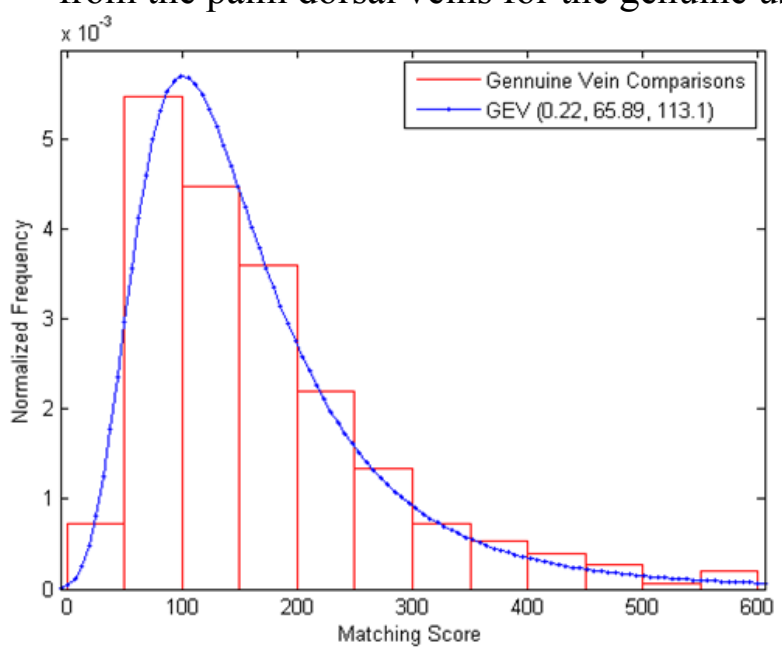

(a)

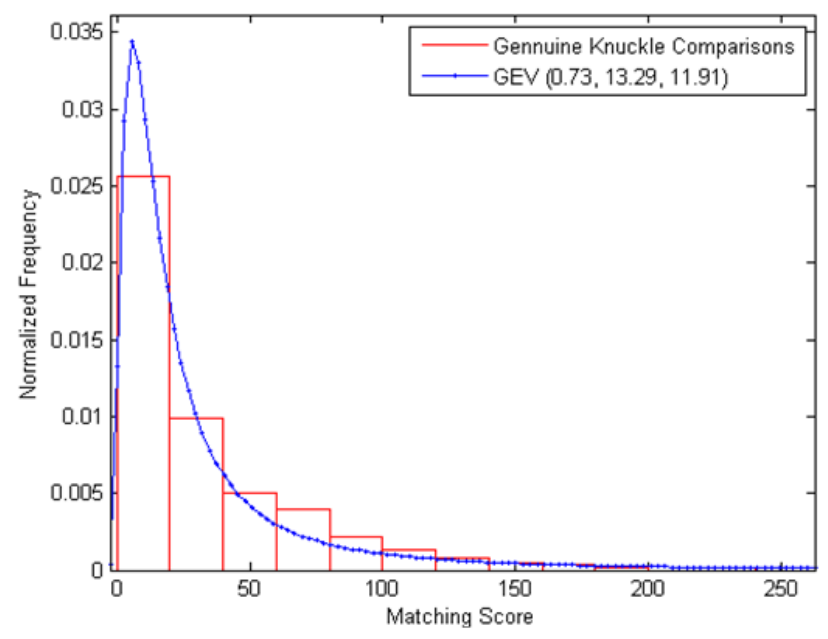

(b)

Figure 15: Estimation of theoretical distribution from (a) genuine vein matching scores and (b) genuine knuckle matching scores 
of genuine user comparison scores obtained from the knuckle tip distances is shown in figure 15 (b). The statistical estimation of confidence level for the biometric authentication requires an excellent match between the theoretical distribution and the real score distribution. Therefore, figure 15 (a)-(b) also illustrate the best fit statistical model obtained for the corresponding score distributions using generalized extreme value distribution. The preference of generalized extreme value (GEV) distribution to model the distribution of real world data has been illustrated in several references [29]-[30]. The GEV distribution was also found to be most appropriate, i.e. best fit, to model the matching score distributions from our experiments. The $\operatorname{GEV}(\mu, \alpha, \tau)$ distribution, for the matching score probability $s$, is characterized by three parameters; $\mu, \alpha$, and $\tau$ representing location, scale and shape respectively. The probability distribution of GEV distribution is characterized as follows:

$$
f\left(s_{i} \mid \tau, \mu, \alpha\right)=\frac{1}{\alpha} \exp \left(-\left(1+\tau \frac{(s-\mu)}{\alpha}\right)^{-\frac{1}{\tau}}\right)\left(1+\tau \frac{(s-\mu)}{\alpha}\right)^{-1-\frac{1}{\tau}} \text { for } 1+\tau \frac{(s-\mu)}{\alpha}>0
$$

The more details on GEV distribution can be found in reference [29]. Daugman [31] has

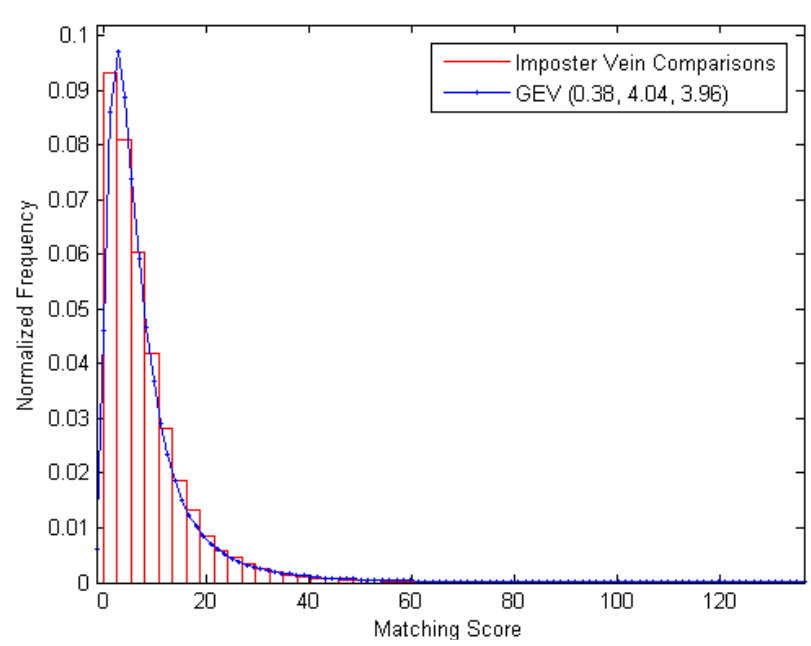

(a)

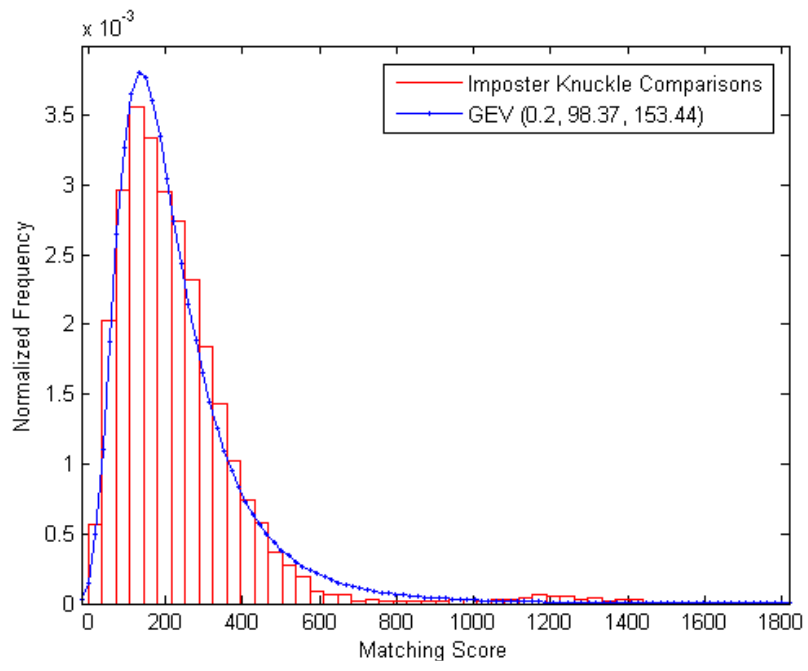

(b)

Figure 16: Estimation of theoretical distribution from (a) imposter vein matching scores and (b) imposter knuckle matching scores 
presented an optimal method for iris representation based on the distribution of a Hamming distances from the irisCode. The binary nature of outcomes from the imposter matching decisions suggests that the Binomial distribution can be more appropriate model for analyzing the distribution of a Hamming distances, generated from the optimal method of iris representation. Instead, the distribution of such matching scores from the palm dorsal veins employed in our work closely follow GEV distribution (figure 15, 16) and suggests its preference (over Binomial or Gaussian) to model the theoretical distribution of matching scores.

\section{Discussion}

The clarity and visibility of subcutaneous blood vessels varies across the user population and depends on the physiological variations, ambient temperature, humidity, hand pose, health and thickness of subcutaneous fat layer. The variation in the size and structure of venous components (e.g. lipid membrane, collagen fibres and nuclei) affect its scattering properties and alters the distribution of photon flux [28]. In addition, the clarity of palm dorsal veins in the acquired images is also influenced by the presence of hairs, moles and scars on skin surface and also its pigmentation. Therefore the investigated approach for the personal authentication does not depend only on the topology of venous network but also on the skin pigmentation and fat depositions on the dorsum of hand. The poor visibility of veins in the acquired images results in inaccurate extraction of venous structure and can generate spurious or missing minutiae points, which degrades the performance of the system. 
The average number of minutiae that that can be extracted from the low quality, i.e. poor vascular visibility and clarity, images were limited. It is interesting to note that the average number of ridge bifurcations extracted per image from our experiments was 2.81 while the average number of ridge endings observed per image was 11.53 . Although the average number of ridge endings is expected to be higher than ridge bifurcations, the effect of associated noise in acquired images was observed to be more pronounced in generating spurious ridge endings than spurious bifurcations. The average number of triplets (table 1 ) of type 1, 2, 3, and 4 per image from our experiments were $1.53,7.32$, 12.84, and 119.82 respectively. The selection of threshold $T_{\mathrm{m}}$ in (5) is a judicious compromise between two key considerations. The larger values of threshold $T_{\mathrm{m}}$ requires large number of triplets to be matched and therefore increases the computational complexity. On the other hand, smaller values of $T_{\mathrm{m}}$ are also not desirable as they limit the genuine matches from the hand vein images with non-local deformations.

\section{Conclusions}

This paper has proposed a new approach for the hand authentication using vein triangulation. In addition, the unique knuckle point perimeter distances were also simultaneously extracted from the same image and utilized to achieve the performance improvement. The utility of knuckle shape biometric has been cited in [9] but there has been negligible attention on its usage in the biometric literature [18]. The experimental results illustrated in previous section, i.e., equal error rate of $1.14 \%$ on the database of 100 users, are certainly promising. The illustrated performance should be interpreted in the context of contactless imaging as such images are expected to present higher intraclass variations as compared to those acquired from fixed imaging using hand docking 
devices. Table 2 presents comparative summary of prior work on the hand vein (back surface) authentication approaches presented in the literature.

Table 2: Comparative summary of related work on hand vein (back surface) based authentication

\begin{tabular}{|c|c|c|c|c|}
\hline Reference & Methodology & Imaging & Database & Performance \\
\hline T. Tanaka and N. Kubo [8] & $\begin{array}{l}\text { FFT based phase } \\
\text { correlation }\end{array}$ & Near Infra Red, HDF & 25 Users & $\begin{array}{l}\text { FAR }-0.73 \% \\
\text { FRR }-4 \%\end{array}$ \\
\hline C.-L. Lin and K.C. Fan [1] & $\begin{array}{l}\text { Multi-resolution } \\
\text { analysis and } \\
\text { combination }\end{array}$ & $\begin{array}{l}\text { Thermal Hand Vein } \\
\text { Imaging }(3.4-5 \mu \mathrm{m})\end{array}$ & 32 Users & $\begin{array}{l}\text { FAR }-1.5 \% \\
\text { FRR }-3.5 \%\end{array}$ \\
\hline $\begin{array}{l}\text { L. Wang and G. Leedham } \\
\text { [2] }\end{array}$ & $\begin{array}{l}\text { Line segment } \\
\text { Hausdorff } \\
\text { distance matching }\end{array}$ & $\begin{array}{l}\text { Thermal Hand Vein } \\
\text { Imaging }(8-14 \mu \mathrm{m})\end{array}$ & 12 Users & $\begin{array}{l}\mathrm{FAR}-0 \% \\
\mathrm{FRR}-0 \%\end{array}$ \\
\hline $\begin{array}{l}\text { Y. Ding, D. Zhuang and } \mathrm{K} \text {. } \\
\text { Wang [13] }\end{array}$ & $\begin{array}{l}\text { Distance between } \\
\text { feature points }\end{array}$ & Near IR Imaging, $\mathrm{HDF}$ & 48 Users & $\begin{array}{l}\text { FAR }-0 \% \\
\text { FRR }-0.9 \%\end{array}$ \\
\hline $\begin{array}{l}\text { J. M. Cross and C. L. Smith } \\
\text { [3] }\end{array}$ & $\begin{array}{l}\text { Sequential } \\
\text { Correlation in } \\
\text { vein maps }\end{array}$ & Near IR Imaging, $\mathrm{HDF}$ & 20 Users & $\begin{array}{l}\text { FAR }-0 \% \\
\text { FRR }-7.9 \%\end{array}$ \\
\hline This Paper & $\begin{array}{l}\text { Matching vein } \\
\text { triangulation and } \\
\text { shape features }\end{array}$ & $\begin{array}{l}\text { Near } \mathbb{R} \text { Imaging, } \\
\text { Contactless }\end{array}$ & 100 Users & $\begin{array}{l}\text { FAR }-1.14 \% \\
\text { FRR }-1.14 \%\end{array}$ \\
\hline
\end{tabular}

* HDF - Hand Docking Frame

This table suggests that the prior approaches have employed small size database but also achieved promising results. The contactless imaging is perceived to be more hygienic and user friendly but it poses problems of missing or spurious vein minutiae points. This is mainly due to the resulting uneven illumination and the fact that the palm back surface is 3D curved surface. The hierarchical weighting of triplets is therefore suggested to achieve better matching performance from such images. The imaging setup employed in our work has been developed for the cooperative users in the indoor environment. It makes an assumption that user is keen to authenticate himself/herself for access and not yet suitable for the uncooperative user. The strong near IR component present in the sunlight also limits the usage of employed imaging setup for outdoor usage. The 
background during the indoor imaging can be controlled and can be conveniently made uniform. Our current imaging setup employs fixed focus and cannot accommodate large changes in the imaging distance. However, inclusion of auto-focus in the imaging setup, similar to as employed in [32] for iris imaging, can help to accommodate large changes in the imaging distance/scale. Although more work remains to be done, our results to date indicate that the combination of hand vein and knuckle shape features constitutes a promising addition to the biometrics based personal authentication. It is encouraging that the performance from such palm-dorsal images, using unconstrained and low-cost image setup, compares very well (or better) with the hand-geometry biometric which is has very high user acceptance [18]. While hand-geometry biometric is highly prone to spoofing, vascular biometric like palm dorsum vein are extremely difficult to forge and at the same time have high user acceptance for its deployment in civilian applications. Further improvement in the performance of proposed system, that simultaneously combines knuckle shape and dorsal vein features, using auto-focus and more sensitive near infrared camera is expected and requires further investigation. The discretization of noisy biometric features has been recently shown to offer significant improvement in the performance. This is especially useful for low-resolution imaging, such as hand geometry or the hand vein employed in this work, and is suggested for further work.

\section{Acknowledgement}

This work was partially supported by the research grant from Ministry of Information and Communication Technology, Government of India, grant no. 12(54)/2006-ESD. The authors would like to thank the anonymous reviewers for their valuable comments and suggestions. 


\section{References}

1. Chih-Lung Lin and Kuo-Chin Fan, "Biometric Verification Using Thermal Images of Palm-Dorsa Vein Patterns" IEEE Trans. Circuits \& Sys. For Video Technology, vol. 14, pp. 199 - 213, Feb. 2004.

2. L. Wang and G. Leedham, "A thermal hand-vein pattern verification system," Pattern Recognition and Image Analysis, S. Singh, M. Singh, C. Apte and P. Perner (Eds), LNCS, vol. 3687, pp. 58-65, Springer, 2005.

3. J. M. Cross and C. L. Smith, "Thermo graphic imaging of the subcutaneous vascular network of the back of the hand for biometric identification," Proc. IEEE $29^{\text {th }}$ Annual Int. Carnahan Conf. Security Technology, Sander-stead, Surrey, pp. 20-35, Oct. 1995.

4. S.-K. Im, H.-M. Park, S.-W. Kim, C.-K. Chung, and H.-S. Choi, "Improved vein pattern extracting algorithm and its implementation," Proc. Int. Conf. Consumer Electronics, pp. 2-3, Jun. 2000.

5. S. K. Im, H. M. Park, Y.W. Kim, S. C. Han, S.W. Kim, and C. H. Hang, "An biometric identification system by extracting hand vein patterns," J. Korean Phys. Soc., vol. 38, pp. 268-272, Mar. 2001.

6. J. Mehnert, J. M. Cross, and C. L. Smith, "Thermal graphic Imaging: Segmentation of the Subcutaneous Vascular Network of the Back of the Hand," Research Report, Edith Cowan University, Australian Institute of Security and Applied Technology, Perth, Western Australia, 1993.

7. S. Z. Li, R. Chu, S. Liao, and L. Zhang, "Illumination invariant face recognition using near-infrared images," IEEE. Tran. Patt. Anal. Mach. Intell., vol. 29, pp. 627639, Apr. 2007.

8. T. Tanaka and N. Kubo, "Biometric authentication by hand vein patterns," Proc. SICE Annual Conference, Yokohama, Japan, pp. 249-253, Aug. 2004.

9. C. Colbert, "Knuckle profile identity verification system," US Patent No. 5,862,246, Jan. 1999.

10. G. T. Park, S. K. Im, and H. S. Choi, "A person identification algorithm utilizing hand vein pattern," Proc. Korea Signal Processing Conference, vol. 10, no. 1, pp. 1107-1110, 1997. 
11. S. Zhao, Y. Wang and Y. Wang, "Biometric verification by extracting hand vein patterns from low-quality images," Proc. $4^{\text {th }}$ Intl. Conf. ICIG, pp. 667-671, Aug. 2007.

12. http://www.viewse.com.cn/ProductOne.asp?ID=106

13. Y. Ding, D. Zhuang and K. Wang, "A study of hand vein recognition method," Proc. IEEE Intl. Conf. Mechatronics \& Automation, Niagara Falls, Canada, pp. 2106 - 2110, Jul. 2005.

14. K. Wang, Y. Zhang, Z. Yuan, and D. Zhuang, "Hand vein recognition based on multi supplemental features of multi-classifier fusion decision," Proc. IEEE Intl. Conf. Mechatronics \& Automation, Luoyang, China, pp. 1790 - 1795, Jun. 2006.

15. L. Wang and G. Leedham, "Near- and Far-Infrared imaging for vein pattern biometrics," Proc. IEEE Intl conf. Video \& Signal based Surveillance, AVSS'06, Sydney, pp. 52-57, Nov. 2006.

16. Handbook of Fingerprint Recognition, D. Maltoni, D. Maio, A. K. Jain and S. Prabhakar, Springer, New York, 2003.

17. S Fantini and M. A. Franceschini, Handbook of Optical Biomedical Diagnostics, SPIE Press, Bellingham, USA, 2002.

18. Handbook of Biometrics, A. K. Jain, P. Flynn, and A. Ross (Eds), Springer, 2007.

19. C. Oden, A. Ercil, and B. Buke, "Combining implicit polynomials and geometric features for hand recognition," Pattern Recognition Letters, vol. 24, pp. 2145-2152, 2003

20. A. Kumar and D. Zhang, "Personal recognition using hand-shape and texture," IEEE Trans. Image Process., vol. 15, no. 8, pp. 2454-2461, Aug. 2006

21. G. Bebis, T. Deaconu, and M. Georgiopoulos, "Fingerprint identification using Delaunay triangulation," Proc. Intl. Conf. Information Intelligence and Systems, pp. 452-459, 1999.

22. L. Wang, G. Leedham and Siu-Yeung Cho, "Minutiae Feature Analysis for Infrared Hand Vein Pattern Biometrics," Pattern Recognition, 41 (3), pp. 920-929, 2008.

23. P. Buddaharaju, I. Pavlidis, P. Tsiamyrtzis, and M. Bazakos, "Physiologybased face recognition in the thermal infrared spectrum," IEEE. Tran. Patt. Anal. Mach. Intell., vol. 29, pp. 613-626, Apr. 2007. 
24. W. Norman, The Anatomy Lesson, http://home.comcast.net/ wnor \{Reproduced with permission

25. O. A. Carretero, "Vascular remodeling and the kallikrein-kinin system," J. Clin. Invest., vol. 115, pp. 588-591, Mar. 2005

26. P Carmeliet, and R. K. Jain, "Angiogenesis in cancer and other diseases," Nature, vol. 407, pp. 249-257, 2000.

27. J.-G. Wang, W.-Y. Yau, A. Suwandy and E. Sung, "Person recognition by palmprint and palm vein images based on 'Laplacianpalm' representation," Pattern Recognition, vol. 41, pp. 1531-1544, 2008.

28. P. S. Pandian, M. Kumaravel, and M. Singh, "Optical imaging and parametric characterization of frostbite changes in human hand tissues," Current Science, vol. 95, no. 2, pp. 196-203, Jul. 2008.

29. S. Kotz and S. Nadarajah, Extreme Value Distributions: Theory and Applications, World Scientific, 2001.

30. S. Coles, An Introduction to Statistical Modeling of Extreme Values, SpringerVerlag, 2001.

31. J. Daugman, "The importance of being random: Statistical principles of iris recognition," Pattern Recognition, vol. 36, no. 2, pp. 279-291, 2003.

32. K. R. Park and J. Kim, "A real-time focusing algorithm for iris recognition camera," IEEE Trans. Sys. Man \& Cybern., Part C, vol. 35, pp. 441-444, Aug. 2005.

33. A. Kumar and K. V. Prathyusha, "Personal authentication using hand vein triangulation," Proc. SPIE Conf Biometric Technology for human identification, vol. 6944, Orlando, pp. 69440E-69440E-13, Mar. 2008.

34. J. Mobley and T. Vo-Dinh, Biomedical Photonics Handbook. CRC Press, 2003.

35. A. K. Jain, Y. Chen, and M. Demirkus, "Pores and Ridges: Fingerprint Matching Using Level 3 Features," IEEE. Tran. Patt. Anal. Mach. Intell., vol. 29, no. 1, pp. $15-27,2007$.

36. http://www.fujitsu.com/global/about/rd/200506palm-vein.html

37. http://www.bionics-k.co.jp/english/product/va200.html

38. R. Derakhshani and A. Ross, "Conjunctival scans for personal identification," $U . S$ Patent No. 7327860, Feb 5, 2008 